\title{
Solar Eruptions and Energetic Particles: An Introduction
}

\author{
N. Gopalswamy ${ }^{1}$, R. Mewaldt ${ }^{2}$, and J. Torsti ${ }^{3}$
}

This introductory article highlights current issues concerning two related phenomena involving mass emission from the Sun: solar eruptions and solar energetic particles. A brief outline of the chapters is provided indicating how the current issues are addressed in the monograph. The sections in this introduction roughly group the chapters dealing with coronal mass ejections (CMEs), solar energetic particles (SEPs), shocks, and space weather. The concluding remarks include a brief summary of outstanding issues that drive current and future research on CMEs and SEPs.

\section{OVERVIEW}

The Sun loses mass in three different ways: the Solar Wind, Coronal Mass Ejections (CMEs), and Solar Energetic Particles (SEPs). These phenomena are signatures of solar variability from the matter point of view; electromagnetic radiation in the form of quiescent and flare emissions represents the other major variability. The mass emissions from the Sun also signal fundamental physical processes such as acceleration of plasmas to high energies, magnetic field reconnection and interaction between large-scale plasma structures. Fast solar wind originates from the open field regions on the Sun known as coronal holes while CMEs originate from the closed field regions such as active regions and filament regions. The interplanetary plasma is the solar wind, and the propagation of CMEs through this plasma represents interaction between them. CMEs often attain super-Alfvenic speeds in the magnetized coronal and interplanetary plasmas and hence are bound to drive fast mode shocks, which in turn are known to accelerate the

\footnotetext{
${ }^{1}$ NASA Goddard Space Flight Center, Greenbelt, Maryland ${ }^{2}$ California Institute of Technology, Pasadena, California

${ }^{3}$ University of Turku, Turku, Finland

Solar Eruptions and Energetic Particles Geophysical Monograph Series 165

This paper is not subject to U.S. copyright. Published in 2006 by the American Geophysical Union 10.1029/165GM02
}

charged particles in the upstream medium to very high energies. Even sub-Alfvenic CMEs interact with the solar wind resulting in momentum exchange described by the aerodynamic drag. Thus, the three types of mass emissions are not independent. Even the flare process is not independent: Almost all CMEs are associated with flares, which accelerate electrons and ions that flow towards the Sun and away from the Sun producing electromagnetic radiation at various wavelengths and injecting flare-heated plasmas into the CMEs. Flares are also associated with the smaller and more frequent SEP events, known as impulsive SEP events. It is thought that impulsive SEP events can provide seed particles for CME-driven shocks causing an interesting mix of SEP compositions and charge states. Studies of these mass outflows from the Sun are also of significant practical importance: they can cause severe space weather effects such as disruption of power systems on Earth, and can pose radiation hazards to our space travelers and spacecraft systems. Various chapters in this volume provide a review of the current status of our understanding of these energetic phenomena, the underlying physics, and why humans care about them.

The concept of mass leaving the Sun was contemplated well over 100 years ago from when high-speed prominence eruptions from the Sun were observed. R.A. Howard describes the historical development culminating in the discovery of CMEs in the early 1970s, and the subsequent milestones in $\mathrm{CME}$ research including the pre-eminent position occupied by CMEs as major drivers of geospace disturbances. The discovery of SEPs preceded that of CMEs by three decades, when Forbush reported the first event. 
$J . R$. Jokipii takes us through the early observations and the development of a theoretical understanding of particle acceleration. He points out the universal nature of the energetic particle distribution, whether it is from the Sun or from the rest of the universe as cosmic rays.

\section{CORONAL MASS EJECTIONS}

S.W. Kahler reviews the current status of CME research drawing heavily from the results of analyzing more than 10,000 CMEs observed by the Solar and Heliospheric Observatory (SOHO) mission. In addition to describing the physical and statistical properties of CMEs, he explains the basic magnetic structures involved in CME initiation and subsequent propagation. He points out that progress in understanding CMEs has been accelerated by the inner coronal imaging in X-rays, EUV and radio, but more observations are needed of spectral lines that can provide information on the physical conditions in CMEs. The outstanding issues concern the magnetic field geometries and topologies of CMEs, and the way in which CME magnetic fields reconnect to become open magnetic fields in the heliosphere. The better sensitivity and extended field of view of the SOHO coronagraphs have resulted in detecting a record number of CMEs. The data size will grow even further when future coronagraphs such as on the STEREO mission observe CMEs with higher cadence. It is therefore necessary to develop automatic techniques for CME detection. E. Robbrecht and D. Berghmans discuss the current status of such techniques for various solar features. R.L. Moore and A.C. Sterling focus on how CMEs are initiated, especially the fast ones that significantly disturb the heliosphere. They identify three fundamental mechanisms that can explain the CME initiation: (1) runaway internal tether-cutting reconnection, (2) runaway external tethercutting reconnection, and (3) ideal MHD instability. The three mechanisms may operate singly or in combination. Magnetograms and movies of chromospheric and coronal features are used to tell which one or which combination of these mechanisms is the trigger for eruption. One of the points they emphasize is the production of a flux rope as a result of the eruption. The flux-rope structure has become a basic entity in CME research, used extensively by CME modelers. Flux-ropes are directly observed in the interplanetary medium as magnetic clouds. The fact that CMEs carry twisted magnetic structures must be related to the removal of accumulated helicity in the corona, as discussed by $A$. Nindos. The study of helicity in CMEs has only started recently, but the importance can be felt in the hemispheric dependence of chirality of filaments, helicity of active regions and the handedness of interplanetary magnetic clouds. R.A. Harrison discusses the connection between flares and CMEs. While opposing views prevail from the flare explosion causing CMEs to flares as a byproduct of CMEs, he points out the common cause for the explosive emission of mass and flare radiation.

I. Roussev and I. Sokolov review current CME modeling efforts, focusing on the flux-rope and shear-arcade models. In particular they discuss the salient features and major weaknesses of the two models. Current observations may not be able to discriminate between the two models. They conclude that future prospects for CME modeling are more optimistic, mainly because of the opportunities the STEREO mission will provide for CME theorists and modelers to answer some of the outstanding issues on CME initiation and propagation.

\section{SEPS AND THEIR RELATION TO FLARES AND CMEs}

The SEPs that reach Earth originate in both flares and CME-driven shocks, and there is on-going debate as to the relative roles of these processes in large SEP events. $T$. von Rosenvinge and H.V. Cane provide an overview of the SEP phenomenon and of our current state of understanding. They focus on the current paradigm of SEP events and suggest that some modification is needed in the two-class (impulsive and gradual SEP events) picture. They conclude that our understanding of the SEP and related phenomena is still not complete despite the extensive body of observations currently available.

R.A. Mewaldt, C.M.S. Cohen, and G.M. Mason provide an authoritative account of the current state of affairs in SEP physics, covering a wide range of topics related to the source material for large SEP events. They review the recent work on the particle sources contributing to the seed population for the accelerated particles. The gradual SEP events differ in composition from the bulk solar wind in several key respects, implying that solar wind does not supply the principal seed population for these events. The suprathermal pool that is accelerated by CME-driven shocks seems to be made up of ions from impulsive solar flares and previous gradual events, CIR events, pickup ions, CME ejecta, and the suprathermal tail of the solar wind.

The ionic charge states of SEPs reflect the thermal history of the material that is accelerated and possible non-thermal processes that may remove electrons. Observations by ACE, SAMPEX and SOHO show that the mean charge state often increases with energy over the energy range from $\sim 50$ $\mathrm{keV} /$ nucleon to $50 \mathrm{MeV} / \mathrm{nucleon}$. M. Popecki reviews these observations and discusses some of the interpretations that have been offered for this unexpected behavior, including mixtures of heated-flare and coronal material, and stripping of electrons during the acceleration and transport of the ions. 
L. Kocharov presents the results of a model of the evolution of the ionic charge states of Fe during acceleration and transport and discusses what can be learned from ionic charge state observations. B. Klecker reviews recent observations of the ion charge state composition in SEP events and discusses the implications for our understanding of different acceleration scenarios. There is considerable progress in our knowledge of the elemental and isotopic composition, energy spectra and ionic charge states in gradual and impulsive SEP events. These new results show that the classification into two distinct types of events was oversimplified, and they provide important new constraints on theoretical models for SEP acceleration and transport.

During Solar Cycle 23 considerable progress in understanding particle acceleration in SEP events resulted from new remote-sensing observations by missions such as RHESSI, SOHO, TRACE and Yohkoh. Some fraction of the particles accelerated in flares escape into interplanetary (IP) space along open field lines, while others, trapped on closed field lines, interact in the chromosphere and photosphere. A. MacKinnon gives an overview of the use of $\mathrm{X}$-rays, $\gamma$-rays and radio observations in obtaining direct information on flare site fast electrons and ions. He points out that observations of the SEPs detected in the IP medium as well as the flare-site populations inferred from radiative diagnostics are ultimately needed to fully understand the eruptive events. G. Share presents RHESSI observations of the gamma-ray and continuum radiation in some of the largest solar events of this past solar maximum, and reviews how these observations can be used to study the composition and energy spectra of particles accelerated in the flare, as well as the composition of the solar atmosphere. One puzzling result is that the proton/alpha ratio derived from the gamma-ray data is considerably smaller than is observed with in situ SEP measurements. M.J. Aschwanden uses data from Yohkoh, SOHO, TRACE, and CGRO to illustrate the magnetic topologies in the accelerating region, the asymmetry of upward versus downward acceleration, and particle access to interplanetary space. Of particular interest are the magnetic topologies that lead to reconnection between open and closed field lines. Along similar lines, R.P. Lin uses recent observations from Wind and RHESSI to study solar electron events observed both in situ at $1 \mathrm{AU}$ and remotely at radio and $\mathrm{x}$-ray wavelengths. While the properties of the interacting and escaping electrons are correlated, the correlation is not explained by simple models.

\section{CME-DRIVEN SHOCKS AND SEPS IN THE HELIOSPHERE}

One of the greatest successes of space physics is the theory of shock acceleration, which has been successfully applied to particle acceleration at shocks ranging from planetary bow shocks and to interplanetary shocks, to supernova shocks. CME-driven shocks accelerate both electrons and ions. The most common signature of shock-accelerated electrons is the radio emission known as type II radio bursts, which can be used to track the shocks from the Sun to all the way to Earth and beyond. Although an additional shock source of flare blast waves is often assumed, $N$. Gopalswamy has provided arguments that the type II phenomenon over the entire SunEarth distance can be explained in terms of CME-driven shocks. He uses the hierarchical relationship between $\mathrm{CME}$ kinetic energy and the wavelength range of type II emission, the weakening of evidence for CME-less type II bursts, the realistic profile of the fast-mode speed in the corona and IP medium, and the close connection between SEP events and type II radio bursts to support his arguments. G. Mann explores the relation between coronal shocks (inferred from coronal type II bursts), Moreton waves, and EUV transient waves (known as EIT waves because of their detection by the Extreme-ultraviolet Imaging Telescope (EIT) on board $\mathrm{SOHO})$. He also extends the Alfven speed profile in the corona to two dimensions and attributes the inability of flaregenerated shocks to propagate beyond the Alfven-speed maximum to explain the metric type II bursts. K.-L. Klein compares the particle acceleration by flares and CME-driven shocks. He calls for a much closer interrelationship between flares and CMEs as sources of SEPs, rather than assuming a clear-cut distinction between flare-related and CME shockrelated SEP events.

$M . A$. Lee and $R$. Vainio review the present status of the theory of accelerating energetic ions at CME-driven shocks. Although the theory can generally account for the observed features of these events, there remain outstanding issues to be addressed, including ion injection into the acceleration process, an improved description of the wave intensity excited by the accelerated particles, and injection and acceleration at quasi-perpendicular shocks. A. Tylka and M.A. Lee investigate the correlations among spectral and compositional characteristics, which serve as powerful constraints for their models of SEP events (both gradual and impulsive). Starting with CME-driven shocks as the accelerator for gradual SEPs, they were able to account for the correlated spectral and compositional variability in SEPs by including the shock normal angle and a compound seed population as variables. On the other hand, they point out the lack of understanding of the spectra and composition of impulsive SEP events in terms of flare parameters, such as size, duration, and magnetic topology. C.M.S. Cohen reviews research on energetic storm particles (ESP) events, which are same as the SEP events but occur when the accelerating shock passes the observing spacecraft. She provides an overview of the observations and theories of ESPs from their first detection to our current understanding. ESP events are testimony to the 


\section{SOLAR ERUPTIONS AND ENERGETIC PARTICLES: AN INTRODUCTION}

shock acceleration process since parameters of the shock can be measured directly. SEP events with particle energy high enough to penetrate Earth's atmosphere are known as ground level enhancement (GLE) events. C. Lopate presents an overview of how ground-based instruments have been used to study GLE events over the past fifty years. Despite of the relative rarity of GLEs, he argues that they are key to our full understanding of the processes involved in high-energy solar phenomena, both on the Sun itself and during the travel through interplanetary space to Earth. The CME-shock vs. flare reconnection debate is also applicable to the acceleration of GLE events. Particles accelerated near the Sun reach Earth by propagating through the dynamical, turbulent solar wind. The paper by $W$. Droege presents the results of a dynamical theory of particle transport in the interplanetary medium that models the evolution of SEP events using transport coefficients derived solely from observed solar-wind plasma observations. The model is successful at reproducing observations from $\mathrm{keV}$ to $\mathrm{GeV}$ energies.

The interplanetary counterparts of CMEs are known as interplanetary CMEs (ICMEs). In addition to driving shocks that can accelerate energetic particles, there are other interplanetary signatures of ICMEs, including bi-directional ion flows, and the capability of ICMEs to confine (or exclude) energetic particles. $D$. Lario reviews observations of the effects of ICMEs on energetic particles from a variety of spacecraft, using archival data extending from inside $1 \mathrm{AU}$ to the outer heliosphere. A. Struminski and B. Heber report on a comparison between two sets of SEP events observed by Ulysses in the heliosphere (a set of eight SEP events in the ecliptic plane around $5 \mathrm{AU}$ and another set of six events at high heliographic latitudes). They compare these events with GOES observations at $1 \mathrm{AU}$ and infer that $>30 \mathrm{MeV}$ SEPs are observed over an angular range of $120^{\circ}$ in both longitude and latitude. They suggest that CME-driven shocks may not be able to account for these events.

\section{SPACE WEATHER}

CMEs can be both geoeffective (CME plasma impacts Earth's magnetosphere producing geomagnetic storms) and SEP-effective (accelerated particles from near the Sun extend far into the heliosphere). It is the very fast, Earthwarddirected CMEs that produce the highest-intensity SEP events at Earth and these same CMEs are often very effective at triggering geomagnetic storms once they reach Earth. CMEdriven shocks reaching Earth are also responsible for the ESP events, which can be a direct radiation hazard for space-based assets. E. Valtonen reviews geoeffective and SEPeffective CMEs and describes how energetic particle observations can be used to identify interplanetary CMEs and shocks approaching Earth. As a feasibility demonstration, he gives two examples illustrating the methods of using energetic particle observations for forecasting geomagnetic storms. He also summarizes the observed geoeffectiveness of halo CMEs and, in particular, magnetic clouds.

During the largest geomagnetic storms SEP ions and electrons may suddenly have access to the inner magnetosphere, where they can become trapped, forming a new radiation belt that stands out against the quiescent trapped particle populations. J.E. Mazur and collaborators present observations of new radiation belts during Solar Cycle 23 that contain SEP heavy ions, and it discuss some of the conditions that determine when such new belts are created, and how long they survive. I. Roth, M.K. Hudson, B.T. Kress, and K.L. Perry survey geomagnetic modifications due to solar/heliospheric drivers, geomagnetic eigen oscillations and the mechanisms of trapped particle dynamics. They explain how sudden deformation of the field configuration of the magnetosphere can cause trapping of SEPs on a drift time scale to form transient proton and heavy ion belts deep in the magnetosphere. They also point to recent observations which have significantly furthered our understanding of solar wind control of the magnetospheric energetic particle environment.

SEPs have recently taken on new importance in space weather as a result of NASA's decision to once again send astronauts beyond the protective cover of Earth's magnetosphere. R.E. Turner reviews the hazards that SEPs could pose to astronauts during transit to the Moon or Mars, and on the lunar surface. He also discusses how research by the space science community can mitigate the effects of SEPs by providing improved forecasts of the onset, evolution, and intensity of large SEP events.

H.E.J. Koskinen and K.E.J. Huttunen provide a critical overview of the worldwide efforts on space weather and explain the complexities in this multidisciplinary research. They emphasize the need for physical understanding of $\mathrm{CME} /$ flare production in solar sources and the propagation of CMEs before developing accurate forecasting. They call for the need to understand the structure of ICMEs (shock, sheath and magnetic cloud) for the prediction of geomagnetic storms and to understand their variability at high and low latitudes. They also identify several key areas in which progress is badly needed: prediction of the final intensity of a given storm, the acceleration of electrons to relativistic energies, and the creation of extremely strong induction effects between the ionosphere and the ground.

\section{CONCLUDING REMARKS}

To conclude, the set of articles presented in this monograph provides an overview of the broad range of issues involved in the generation of solar eruptions and energetic 
particles and how they propagate to Earth and other destinations in the heliosphere. The rapid progress made in the recent years has been possible thanks to a new generation of instruments and theoretical models, as well as to the ready availability of extended and uniform data sets from various ground and space-based data sources. Yet, there is much more to be done. Some of the key drivers of current research and areas that need more work can be summarized as follows:

The uniform and extended observations by the SOHO mission have revealed that only the fastest of the $10000+$ CMEs are important for heliospheric impact. Most of the current numerical simulation efforts can deal with only slow CMEs. What is needed is the development of realistic models of fast CMEs. Recent results on the composition and charge state of material in ICMEs has brought out an undeniable link between the flare process and CME initiation. This connection needs to be pursued to understand the formation and final configuration of CMEs in interplanetary space.

Predicting the existence and magnitude of the southward magnetic field component of ICMEs based on measurements near the solar surface is a difficult but a crucial step in understanding the complete structure of CMEs. Future missions such as STEREO and Solar-B are likely to provide key information towards such an understanding of the magnetic properties of CMEs near the Sun. A related issue is the magnetic structure of ICMEs: one view is that all ICMEs are magnetic clouds and the differences between them arise from the vantage point of the observation. Proving or disproving this view will go a long way toward validating numerical models of CMEs, most of which have a flux-rope structure as the basic ingredient. With STEREO, supplemented by near-Earth observations, 'there will be multipoint in situ measurements of CME structure in addition to STEREO images.

One characteristic of SEP-associated CMEs is their ability to drive shocks. Shocks ahead of geoeffective CMEs also mark the sudden commencement of geomagnetic storms. Radio dynamic spectra of type II bursts are the only way to remotely sense the shocks when they are still near the Sun. It is highly desirable that white-light signatures of shocks are identified corresponding to the times of type II bursts.

As for SEPs, understanding the deviation from the familiar two-class picture (impulsive and gradual events) is very much needed. While there is no question of particle acceleration by CME-driven shocks (e.g., the ESP events and type II radio bursts), what we lack is a realistic assessment of the relative importance of flare and CME related SEP events. Multi-point measurements by STEREO should make it possible to separate the contributions from these two acceleration processes. Another issue closely related to the two-class scenario is the nature of the source material accelerated by CME-driven shocks. A proper understanding of the relation between SEPs and the progenitor CMEs is crucial for predicting the intensity, duration, and composition of SEPs in the interplanetary medium.

There is a spread of orders of magnitude in the intensity of solar particles that result from CME-driven shocks of any given shock velocity. Are these differences due to shock geometry (quasi-perpendicular vs. quasi-parallel), to differences in wave activity, to pre-conditioning by previous CMEs, or to differences in the seed-particle populations? To understand why some shocks are more efficient accelerators than others can best be answered by future missions that will send spacecraft closer to the Sun, including the ESA Solar Orbiter mission (a single spacecraft to $0.22 \mathrm{AU}$ ), the Inner Heliospheric Sentinels (4 spacecraft in orbit between 0.25 and $0.72 \mathrm{AU}$ ) and Solar Probe (a single spacecraft to traverse the solar corona at a distance of 4 solar radii from the Sun). These exploratory observations, supplemented by remote sensing data, should form the basis for theoretical models that can forecast the onset and evolution of SEP events with much greater accuracy. 